\title{
Coexistence de 2 formes de phénolsulfotransférase dans le rein humain et les plaquettes sanguines
}

\author{
Sylvain Demassieux, Yveline Romain, \\ Serge Carrière
}

\section{Summary}

Phenolsulfotransferase

(EC

2.8.2.1. PST) catalyzes sulfation of phenolic xenobiọtic drugs and endogenous phenolic amines like catecholamines (CA). Since sulfated CA may not represent only excretion products but also alternate substrates in CA metabolism, the sulfoconjugating system in human kidney was studied and compared with that previously demonstrated in platelets. Two components with PST activity (PST I and II) were separated and purified by differential sedimentation, ammonium sulfate fractionation and ion-exchange and affinity chromatographies from either platelets or kidney homogenates. PST I from either tissue catalyzes sulfation of p-nitrophenol, while PST II catalyses that of CA. At $1 \mu \mathrm{M}, 2,6$ dichloro- $p$ -

\section{ADRESSE}

S. Demassieux, Y, Romain, S. Carrière : Centre de Recherches Maisonneuve-Rosemont, 5415 Bd. l'Assomption, Montréal, Québec, $\mathrm{HrT}_{2} \mathrm{M}_{4}$, Canada et Département de physiologie, Université de Montréal, Case Posmle 6128, Suc-

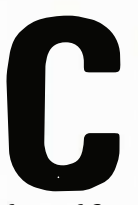
hez l'homme, la majeure partie des catécholamines présentes en circulation se trouvent sous la forme de dérivés sulfatés, si bien que la sulfoconjugaison de ces molécules biosynthétiques a été considérée comme une voie métabolique aboutissant à la formation de composés inactifs destinés à l'excrétion. Des expériences récentes ont cependant permis de montrer que les dérivés sulfoconjugués de la dopamine peuvent régénérer la dopamine libre à la faveur d'une hydrolyse catalysée par des arylsulfate sulfohydrolases (ARS) $[1,2]$, constituer des intermédiaires métaboliques actifs dans la synthèse d'autres catécholamines $[2,3,4]$, ou encore avoir leurs propres effets physiologiques [5].

C'est la phénolsulfotransférase (PST) qui catalyse la sulfatation des phénols en présence du cofacteur, donneur de sulfate, le $3^{\prime}$-phosphoadénosine $\quad 5$-phosphosulfate (PAPS). Chez l'homme, des activités PST ont été décelées dans de nombreux tissus et récemment deux PST, aux caractéristiques physicochimiques différentes, ont été séparées l'une de l'autre à partir des plaquettes sanguines humaines $[6$, 7]. L'une d'elle possède une affinité marquée pour la dopamine $[7,8]$, précurseur biosynthétique direct de la noradrénaline et de l'adrénaline.
Étant donné l'existence du rôle important joué par la dopamine libre comme facteur natriurétique et comme agent vasoactif au niveau rénal [9] et la présence de quantités importantes de dopamine sulfoconjuguée en circulation, il est important de caractériser au niveau du rein chez l'homme, une PST capable de sulfater la dopamine. Cette enzyme constituerait, avec les ARS déjà présentes dans le rein humain (résultats non publiés), un système enzymatique susceptible d'ajuster les taux de dopamine libre et sulfoconjuguée aux besoins de l'organisme. Outre la mise en évidence dans le tissu rénal humain d'une enzyme dont les caractéristiques seraient identiques à celles de l'enzyme plaquettaire qui sulfate la dopamine, la mesure de l'activité PST plaquettaire dans différentes situations physio-pathologiques pourrait constituer une estimation simple de cette même activité dans le rein, voire dans d'autres tissus de l'organisme.

L'activité PST est évaluée à l'aide d'un essai radioenzymatique basé sur la mesure du transfert du groupement sulfate marqué au ${ }^{35} \mathrm{~S}$ du PAPS sur un substrat phénolique [10]. Les reins provenant d'un donneur sain décédé d'un polytraumatisme à la suite d'un accident de la route, ont été originellement prélevés pour une transplantation, mais 


\section{Summary}

nitrophenol (DCNP) almost completely inhibits $p$-nitrophenol sulfation by PST I while sulfation of dopamine by PST II remains fully active. With respect to their behaviour on ionexchange and affinity columns, sensitivity to DCNP inhibition and to substrate specificity, PST I and PST II represent two distinct proteins both present in platelets and kidneys in the human being. The use of $p$-nitrophenol and dopamine represents an unequivocal way to distinguish PST I from II activity in platelets which might be representative of sulfate conjugation of p-nitrophenol and CA elsewhere in the body.

n'ont pas été utilisés à cette fin à cause de malformations anatomiques. Les plaquettes sanguines provenant de donneurs sains (20-40 ans) sont isolées et purifiées par sédimentation différentielle [11]. Les reins et les plaquettes sont homogénéisés dans une solution isotonique tamponnée à $\mathrm{pH} 7,4$.

A partir des homogénats ainsi obtenus, les méthodes de purification des PST plaquettaires et rénales comprennent successivement une sédimentation différentielle, un fractionnement par le sulfate d'ammonium, une chromatographie sur une colonne échangeuse d'anions (DEAE-Sepharose ${ }^{\circledR}$ ou DEAESephacel ${ }^{\circledR}$ ) et entın une chromatographie sur Blue-Sepharose ${ }^{\circledR}$.

\section{Purification des PST}

La figure 1 , ci-contre, montre que les chromatographies sur DEAESepharose et sur DEAE-Sephacel ont permis de discriminer physiquement deux formes de PST dans le rein et les plaquettes sanguines.

$m / s n^{\circ} 6$ octobre 85
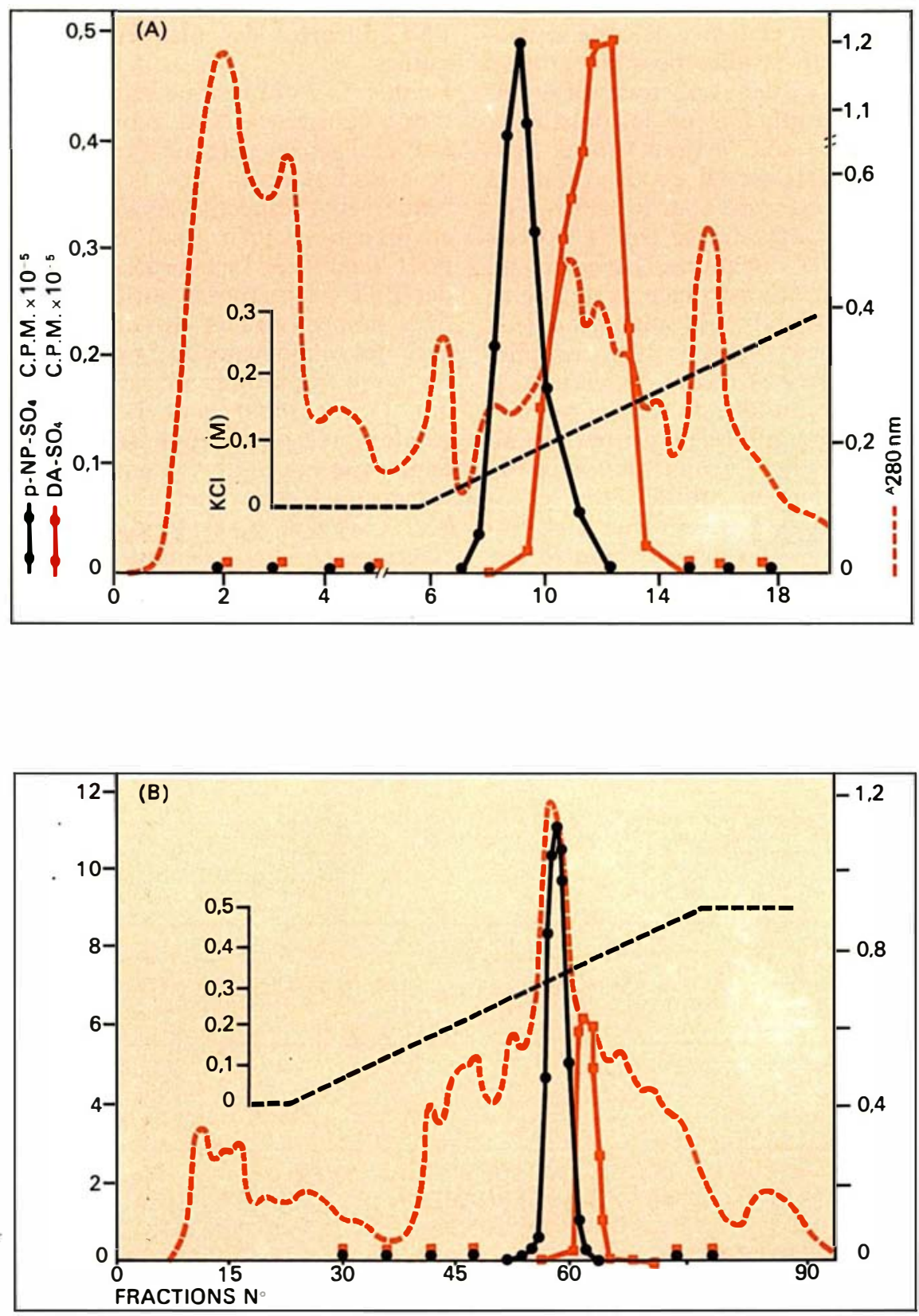

Figure I. Séparation des deux formes de PST plaquettaires et rénales. (A) Le surnageant $105000 \mathrm{~g}$ d'un homogénat de plaquettes qui proviennent de $400 \mathrm{ml}$ de sang est chromatographié sur une colonne $(26,5 \times 1,5 \mathrm{~cm})$ de DEAE-Sephacel. Les protéines sont éluées par un tampon phosphate $20 \mathrm{mM}$ à $\mathrm{pH} 8,0$, puis par un gradient de $\mathrm{KCl}$ dans ce même tampon. (B) Le surnageant $105000 \mathrm{~g}$ d'un homogénat de rein humain $(63 \mathrm{~g})$ est soumis $\dot{a}$ un fractionnement par le $\left(\mathrm{NH}_{4}\right)_{2} \mathrm{SO}_{4}$. La fraction protéique qui précipite entre 30 et $60 \%$ de saturation est chromatographiée sur une colonne $(38 \times 2,5 \mathrm{~cm})$ de $D E A E-S e p h a r o s e$. L'élution des protéines est réalisée avec un tampon phosphate $20 \mathrm{mM}$ à pH 8,0, puis par un gradient de $K \mathrm{Cl}$ dans ce même tampon. L'activité PST I est évaluée avec le p-nitrophénol $4 \mu M(p-n P$, O) et l'activité PST II avec la dopamine $50 \mu M(D A, \square)$. L'ordonnée représente l'activité PST exprimée en C.P.M./min. d'incubation à $35^{\circ} \mathrm{C}$ en présence de $50 \mu L$ de chaque fraction. Les protéines éluées sont estimées par l'absorbance à $280 \mathrm{~nm}\left(A_{280} \mathrm{~nm}\right)$. 
La mise en évidence de cette séparation a été rendue possible grâce à l'emploi de deux substrats différents le $p$-nitrophénol et la dopamine. Quel que soit le tissu utilisé, l'enzyme qui est éluée la première (PST I) est active sur le $p$-nitrophénol mais ne catalyse pas la sulfatation de la dopamine. Inversement, l'enzyme qui est éluée la deuxième (PST II) catalyse la sulfatation de la dopamine et non celle du p-nitrophénol ( figure 1 ). Afin de vérifier si cette similitude de comportement chromatographique n'est pas due au hasard, nous avons poursuivi la purification en utilisant une chromatographie d'affinité sur Blue-Sepharose ${ }^{\circledR}$. La figure 2 montre que les PST rénales se lient fortement au Blue-Sepharose. En effet la PST II et la PST I ne sont respectivement décrochées que pour des concentrations en $\mathrm{KCl}$ aussi élevées que $0,55 \mathrm{M}$ et $0,75 \mathrm{M}$. Nous observons un résultat identique avec les
PST purifiées des plaquettes sanguines.

Le tableau I qui résume les résultats d'une expérience type, montre que la PST I rénale a été purifiée 90 fois et la PST II rénale 37 fois avec des rendements respectifs de 30 et $4 \%$ en prenant le surnageant $105000 \mathrm{~g}$ pour référence. Les formes I et II des PST plaquettaires ont été purifiées respectivement 40 et 50 fois avec des rendements de 15 et $55 \%$. La perte importante de rendement que l'on observe pour la PST II rénale après purification sur BlueSepharose (32 à 3,8\%) comparativement à celle obtenue pour la PST I (87 à $30 \%)$ est l'indice d'une cinétique rapide de dénaturation de la PST II. En effet, la PST I rénale a été chromatographiée sur BlueSepharose immédiatement après la chromatographie sur DEAE-Sepharose, la PST II étant gardée à $4^{\circ} \mathrm{C}$ en solution. A l'inverse, nous avons purifié la PST II plaquettaire avant la PST I. En conséquence, la perte de rendement observée pour la PST I plaquettaire (43 à $15 \%$ ) est négligeable pour la PST II. Compte tenu des quantités de matériel utilisé pour la purification, le tableau I montre aussi que le rein contient plus de PST I et de PST II que les plaquettes sanguines.

\section{Caractéristiques physico-chimiques}

La cinétique de dénaturation des deux formes purifiées de PST, aussi bien d'origine rénale que plaquettaire, est rapide. Aussi, pour l'étude de leur affinité envers la dopamine et le $p$-nitrophénol et l'étude de leur sensibilité envers le 2,6-dichloro- $p$ nitrophénol (DCNP), un inhibiteur reconnu de la sulfoconjugaison [12], nous avons utilisé des fractions lyophylisées obtenues après chromatographie sur DEAE-Sephacel ou DEAE-Sepharose.

\begin{tabular}{|c|c|c|c|c|c|c|c|}
\hline \multicolumn{8}{|c|}{ Tableau I } \\
\hline & & étape & $\begin{array}{l}\text { protéine } \\
(\mathrm{mg})\end{array}$ & $\begin{array}{c}\text { activité } \\
\text { spécifique } \\
\text { (unité/mg) }\end{array}$ & $\begin{array}{l}\text { activité } \\
\text { totale } \\
\text { (unité) }\end{array}$ & $\begin{array}{c}\text { facteur } \\
\text { de } \\
\text { purification }\end{array}$ & $\begin{array}{c}\text { rendement } \\
(\%)\end{array}$ \\
\hline \multirow{2}{*}{ plaquettes } & 1 & $\begin{array}{c}100000 \mathrm{~g} \\
\text { DEAE:SEPHACEL } \\
\text { BLUE-SEPHAROSE }\end{array}$ & $\begin{array}{l}81 \\
7,1 \\
0,3\end{array}$ & $\begin{array}{r}30 \\
150 \\
1250\end{array}$ & $\begin{array}{r}2430 \\
1060 \\
370\end{array}$ & $\begin{array}{r}1 \\
5 \\
40\end{array}$ & $\begin{array}{r}100 \\
43 \\
15\end{array}$ \\
\hline & II & $\begin{array}{c}100000 \mathrm{~g} \\
\text { DEAE-SEPHACEL } \\
\text { BLUE-SEPHAROSE }\end{array}$ & $\begin{array}{r}81 \\
8,3 \\
0,9\end{array}$ & $\begin{array}{r}82 \\
446 \\
4300\end{array}$ & $\begin{array}{l}6642 \\
3680 \\
3650\end{array}$ & $\begin{array}{l}1 \\
5,4 \\
52\end{array}$ & $\begin{array}{r}100 \\
55 \\
55\end{array}$ \\
\hline \multirow{2}{*}{ rein } & I & $\begin{array}{c}100000 \mathrm{~g} \\
\left(\mathrm{NH}_{4}\right)_{2} \mathrm{SO}_{4} \\
\text { DEAE-SEPHAROSE } \\
\text { BLUE-SEPHAROSE }\end{array}$ & $\begin{array}{r}1370 \\
716 \\
106 \\
4,5\end{array}$ & $\begin{array}{r}22 \\
46 \\
147 \\
2010\end{array}$ & $\begin{array}{r}30100 \\
32950 \\
26180 \\
9050\end{array}$ & $\begin{array}{c}1 \\
2,1 \\
11,2 \\
91\end{array}$ & $\begin{array}{r}100 \\
109 \\
87 \\
30\end{array}$ \\
\hline & 11 & $\begin{array}{c}100000 \mathrm{~g} \\
\left(\mathrm{NH}_{4}\right)_{2} \mathrm{SO}_{4} \\
\text { DEAE-SEPHAROSE } \\
\text { BLUE-SEPHAROSE }\end{array}$ & $\begin{array}{l}1370 \\
716 \\
34 \\
1,42\end{array}$ & $\begin{array}{l}18,6 \\
15,2 \\
238 \\
690\end{array}$ & $\begin{array}{r}15500 \\
10900 \\
8100 \\
980\end{array}$ & $\begin{array}{l}1 \\
0,82 \\
12,8 \\
37\end{array}$ & $\begin{array}{c}100 \\
43 \\
32 \\
3,8\end{array}$ \\
\hline
\end{tabular}

Tableau I. L'unité d'activité spécifique représente la quantité d'enzyme présente dans I mg de protéines et nécessaire à la sulfatation de I pmol de substrat par minute d'incubation à $35^{\circ} \mathrm{C}$. La PSTI est évaluée avec le p-nitrophénol $4 \mu M$ et la PST II avec la dopamine $50 \mu \mathrm{M}$. La concentration en protéines des échantillons est évaluée par la méthode du biuret ou par spectrophotométrie différentielle dans lultraviolet. Ce tableau résume les résultats d'une expérience type. Le matériel de départ est constitué par les plaquettes sanguines provenant de $400 \mathrm{ml}$ de sang et par la moitié environ (63 g) d'un rein humain. 

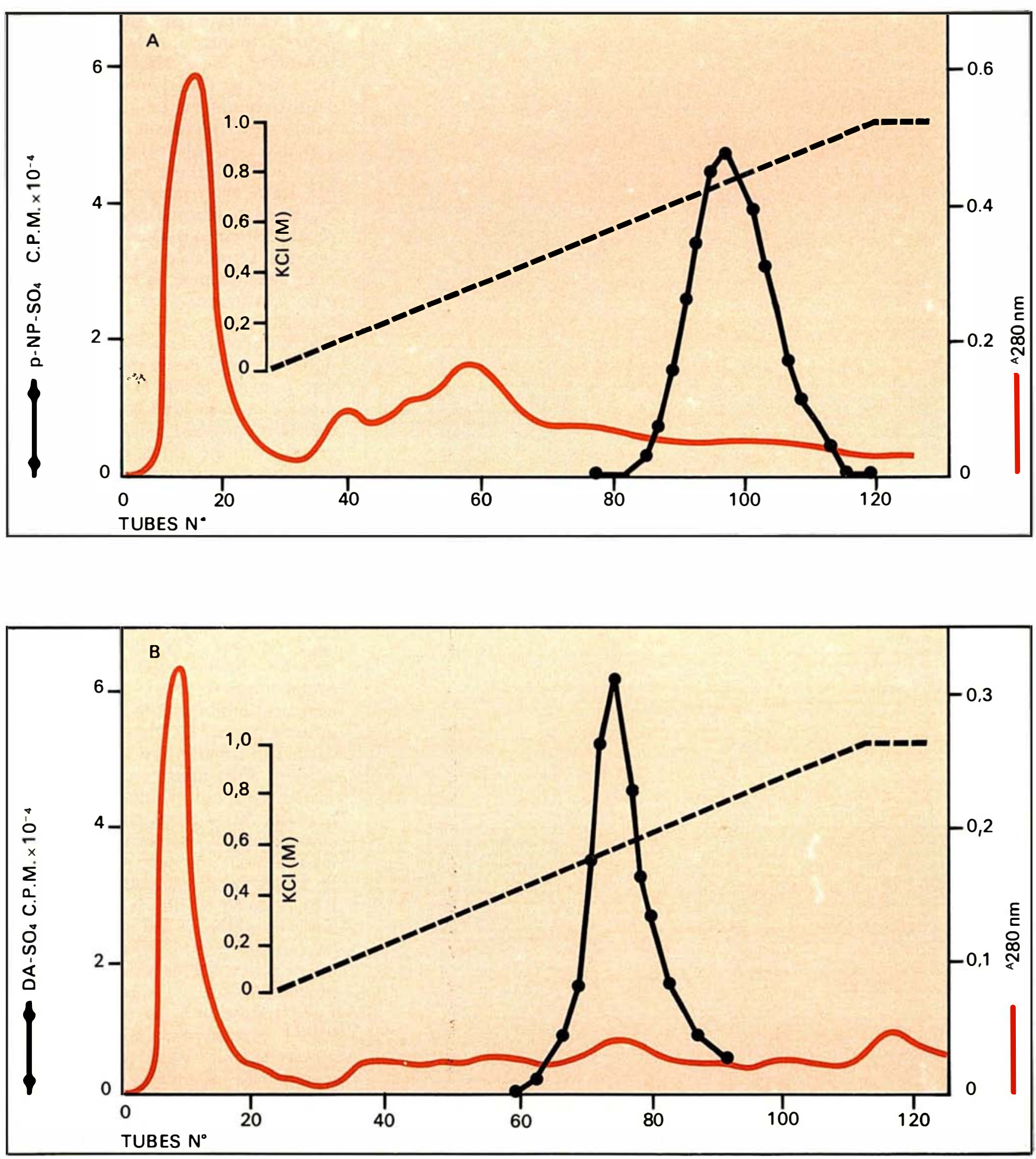

Figure 2. Profïls d'élution de la PST I rénale $(A)$ et de la PST II rénale $(B)$. Les fractions pourvues des activités PST I et PST II obtenues après chromatographie sur DEAE-Sepharose (voir figure I) ont été groupées puis concentrées par ultrafiltration et chromatographiées séparément sur une colonne $(22 \times I \mathrm{~cm})$ de Blue-Sepharose. Les protéines sont éluées par un tampon phosphate $20 \mathrm{mM} \dot{a} \mathrm{pH} 7,4$, puis par un gradient de $K C l$ dans ce même tampon. L'activité enzymatique exprimée en C.P.M.|min. d'incubation $\grave{a} 35^{\circ} \mathrm{C}$ est mesurée en présence de $50 \mu L$ de chaque fraction préalablement dialysée dans un tampon phosphate $20 \mathrm{mM}$ à $p H$ 7,4 avec le p-nitrophénol $(A)$ ou la dopamine (B) comme substrats. Dans les mêmes conditions expérimentales, les comportements chromatographiques des PST I et II plaquettaires sont identiques à ceux observés ci-dessus. 

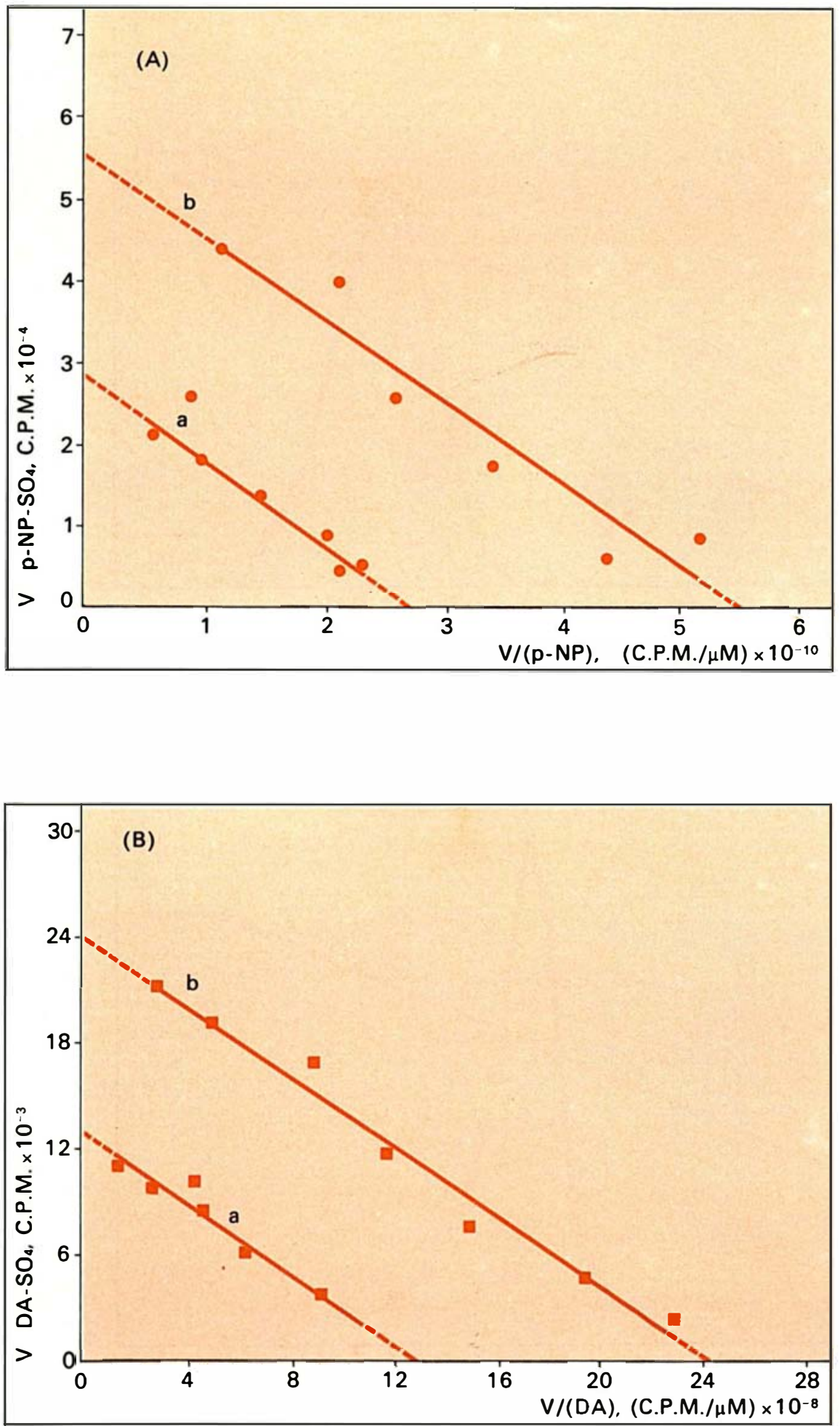

Figure 3. Détermination des constantes apparentes de Michaelis-Menten du p-nitrophénol pour la PSTI rénale $(A)$ et de la dopamine pour la PST II rénale $(B)$. Les Pools I et II lyophilisés obtenus après chromatographie sur DEAESepharose ont été utilisés comme source d'enzyme. La quantité d'enzyme utilisée en $b$ est le double de celle utilisée en a. Dans ce système de coordonnées de Eadie-Hofstee, a) Spécificité de substrats. La figure 3 montre que la valeur de la constante de Michaelis-Menten $\left(K_{m}\right)$ de la PST I rénale pour le $p$-nitrophénol est de $1,1 \mu \mathrm{M}$. Cette valeur est identique à celle de $1,25 \mu \mathrm{M}$ que nous avons pu calculer pour la PST I d'origine plaquettaire [7]. La figure 3 montre aussi que le $\mathrm{K}_{m}$ de la dopamine pour la PST II rénale est de $9,9 \mu \mathrm{M}$. Cette valeur est très semblable à celle que l'on peut calculer pour la PST II, d'origine plaquettaire et qui est de $6,1 \mu \mathrm{M}$ ( figure 4).

Parmi les catécholamines, c'est la dopamine qui est le meilleur substrat pour la forme II. La figure 4 montre en effet que la PST II plaquettaire possède à la fois la plus grande affinité (plus petit $K_{m}$ ) et également la plus grande vitesse de sulfation $\left(\mathrm{V}_{\max }\right.$ relative la plus grande).

b) Inhibition par le 2,6-dichloro-p-nitrophénol. Mulder et Scholtens [12] ont montré que dans le foie de rat la sulfoconjugaison était inhibée par le 2,6-dichloro-p-nitrophénol (DCNP). Nous avons observé que dans le rein humain l'inhibition de la sulfatation induite par le DCNP était dépendante du substrat. Nous avons donc étudié l'effet inhibiteur du DCNP sur les PST I et II purifiées du rein humain. Nous avons trouvé que les constantes d'inhibition $\mathbf{K}_{i}$ du DCNP sont de $0,27 \mu \mathrm{M}$ pour la PST I et de $28 \mu \mathrm{M}$ pour la PST II. Une concentration de $1,0 \mu \mathrm{M}$ en DCNP inhibe la PST I de $95 \%$, tandis que la PST II reste pleinement active. Nous avons rapporté précédemment [7] que le DCNP $1 \mu \mathrm{M}$ inhibe presque totalement la PST I plaquettaire, la sulfoconjugaison de la dopamine par la PST II n'étant pas ou peu affectée.

En conclusion, cette étude démontre qu'il existe dans les plaquettes sanguines humaines au moins deux formes de PST (I et II) qui ont été physiquement séparées grâce à deux méthodes chromatographiques différentes. Les techniques de purification utilisées, les études de spécificité envers la dopamine et le $p$-nitrophénol, et les études d'inhibition induite par le DCNP, nous permettent de conclure que les PST I et II 
plaquettaires sont respectivement identiques aux formes plaquettaires thermostable (TS) et thermolabile (TL) récemment résolues par Reiter et coll. [6]. Sur la base de leurs caractéristiques physicochimiques également, la PST I et la PST II représentent respectivement la forme $P$ (pour Phénol) et la forme $M$ (pour Monoamines) décrites par Rein et coll. dans des homogénats plaquettaires [13].

Nous apportons en outre la démonstration que les deux formes plaquettaires existent également dans le rein humain. L'activité de la PST II qui sulfate les catécholamines, et principalement la dopamine, peut être évaluée sans équivoque dans les plaquettes sanguines entières, tissu aisément accessible, et peut être représentative de la capacité de la sulfatation de la dopamine dans d'autres tissus. Compte tenu de l'existence d'une synthèse de novo de la dopamine au niveau du rein [14] et de son rôle comme facteur vasoactif et natriurétique, la PST II rénale pourrait constituer avec les ARS un système enzymatique susceptible de moduler le rapport dopamine libre/dopamine sulfoconjuguée et contribuer ainsi à l'équilibre hydrosodé de cet organe.

Remerciements. Nous voudrions remercier Madame Ilona Schmidt pour Texcellence de son travail dans la préparation du manuscrit. Ce travail a été rendu possible grâce à une subvention du Conseil National de Recherches du Canada $(M T-3$ I 34$)$.

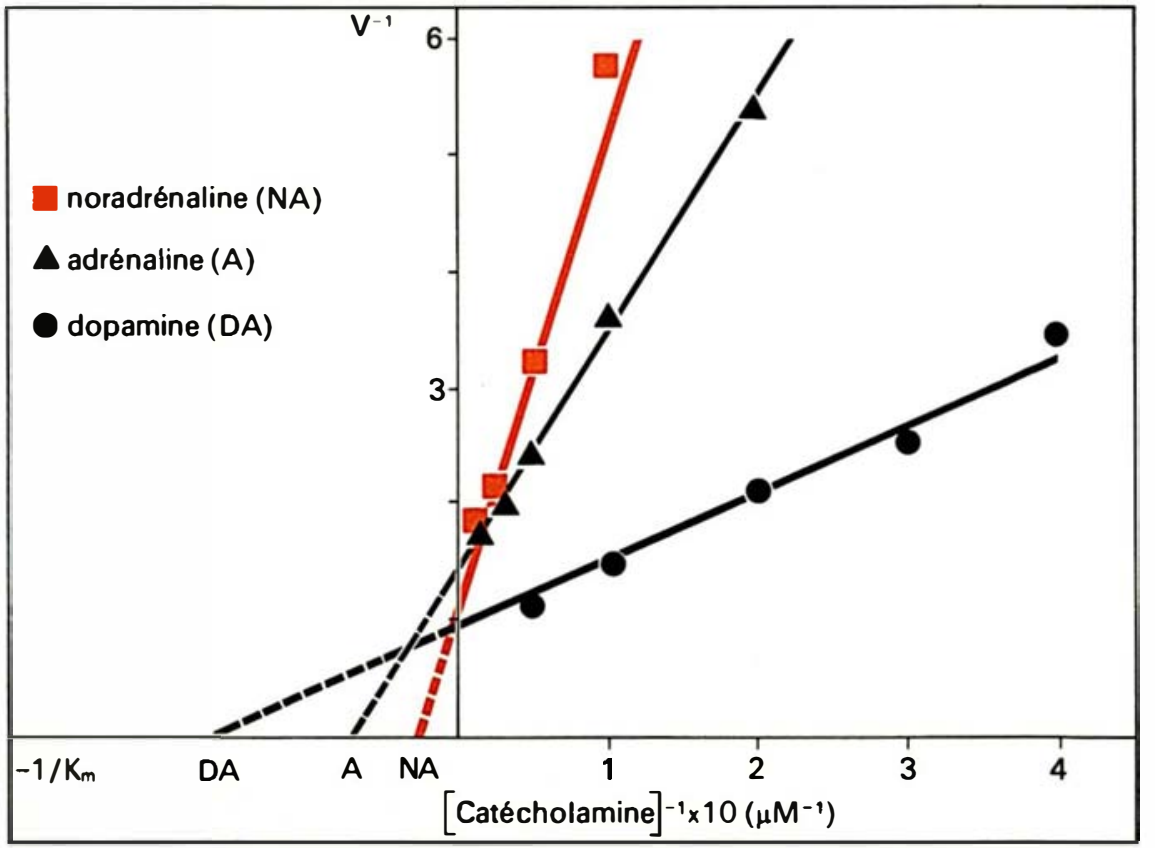

Figure 4. Détermination des constantes apparentes de Michaelis-Menten de la PST II plaquettaire pour les catécholamines. Une même quantité d'enzyme provenant du Pool II obtenu après chromatographie sur DEAE-Sephacel a été utilisée pour déterminer les $\mathrm{K}_{m}$ des trois catécholamines. L'ordonnée représente Pinverse de la vitesse de sulfatation exprimée en valeur relative.

$\mathrm{m} / \mathrm{s} n^{\circ} 6$ octobre 8

\section{REFERENCES}

I. Merits I. Formation and metabolism of ${ }^{14} \mathrm{C}$ dopamine 3 -a-sulfate in dog, rat and guinea pig. Biochem Pharmacol 1976; $25: 829-33$.

2. Buu NT, Nair G, Kuchel O, Genest J. The extra-adrenal synthesis of epinephrine in rats - possible involvement of dopamine sulfate. 7 Lab Clin Med 1981; 98 : 527-35.

3. Buu NT, Kuchel O. The direct conversion of dopamine 3-0-sulfate to norepinephrine by dopamine- $\beta$-hydroxylase. Life $S c i$ 1979; 24 : 783-90.

4. Buu NT, Kuchel O. Dopamine-4-0-sulf ate -a possible precursor of free norepinephrine. Can 7 Biochem 1979; 57 : 11 59-62.

5. Buu NT, Duhaime J, Kuchel O, Genest J. The convulsine effects of dopamine sulfate conjugates in rat brain. Life Sci 1981; 29 : 2311-6.

6. Reiter C, Mwaluko G, Dunette J, Van Loon J, Weinshilboum RM. Thermolabile and thermostable human platelet phenol sulfotransferasesubstrate specificity and physical separation Naunyn Schmiedebergs Arch Pharmacol 1983; 324:140-7.

7. Romain $Y$, Demassieux S, Carrière S. Isolation of two forms of human platelet phenolsulfotransferase (PST). Fed Proc 1983; 42 : 379.

8. Rein G, Glover V, Sandler M. Multiple forms of phenolsulphotransf erase in human tissues - selective inhibition by dichloronitrophenol. Biochem Pharmacol 1982; 31 : 1893-7.

9. Lee MR. Dopamine and the kidney. Clin $S_{C i}$ 1982; $62: 439-48$.

10. Romain Y, Demassieux S, Carriere S. Partial purification and characterization of two isoenzymes involved in the sulfurylation of catecholamines. Biochem Biophys Res Commun 1982; 106 : 999-1005.

I1. Baenzinger NC, Majerus PW. Isolation of human platelets and platelet surface membranes. In: SP Colowick, NO Kaplan, eds. Methods in Enzymology Vol XXXI. New York, San Francisco, London : Academic Press, 1974 : 149-56.

12. Mulder GJ, Scholtens E. Phenolsulphotransferase and uridine diphosphate glucuronyltransferase from rat liver in vivo and in vitro-2,6 dichloro-4-nitrophenol as selective inhibitor of sulphation. 'Biochem 7 1977; 165 : $553-9$

13. Rein G, Glover V, Sandler M. Phenolsulphotransferase in human tissues - Evidence for multiple forms. In: Sandler M, Urdin E, eds. Phenolsulphotransferase in Mental Health Research. Basingstoke: MacMillan, 1981 : 98-1 26.

14. Baines AD, Drangova R. Dopamine production by the isolated perfused rat kidney. Can 7 Physiol Pharmacol 1984; 62 : 272-6.

\section{TIRES A PART}

S. Demassieux : Centre de Recherches Maisonneuve-Rosemont, 5415 Bd. l'Assomption Montréal, Québec, $\mathrm{H}_{\mathrm{I}} \mathrm{T}{ }_{2} \mathrm{M}_{4}$, Canada 